\title{
Screen Reader for Windows Based on Speech Output
}

\author{
Paolo Graziani ${ }^{1}$ and Bruno Breschi ${ }^{2}$ \\ 1 - I.R.O.E. "Nello Carrara" - C.N.R., Via Panciatichi 64 \\ I-50127 Firenze \\ 2 - IDEA I.S.E. s.n.c., Via S. Francesco d'Assisi 65 \\ I-50046 Poggio a Caiano (PO)
}

\begin{abstract}
A so-called "screen reader", enabling blind persons to access the Windows environment, is presented and discussed. It is based on synthetic speech used to present information related to the graphic user interface and organized into a database. Some remarks will be made concerning the comparison between Braille and speech presentation.
\end{abstract}

\section{Introduction}

Transitory Braille and synthetic speech represent the alternative displays for blind people in accessing personal computers.

These two modalities present different characteristics, their advantages and disadvantages have been topics of much discussion. According to a widely accepted opinion, Braille presentation is considered more powerful and preferred by experienced Braille readers, while speech output should be reserved to blind persons who have difficulty in reading Braille.

Another criterion of choice would be represented by the cost of these technologies: a Braille display costs about ten times that of a screen reader based on speech output. This consideration would also lead to the conclusion that those who choose the speech synthesis are forced into doing so for economical reasons.

By analyzing this problem, this classification of blind computer users and of performances of technical aids does not appear entirely accurate. In fact, we can find a number of blind persons who prefer speech synthesis even though they are good Braille readers since, once they become practiced users, they appreciate the superior possibilities of effectiveness in interacting with the machine, and their hands free from reading tasks.

The initial preference of a Braille display has to be considered a prejudice caused by greater familiarity with this modality and the consequently easier approach to the machine by means of this method. The speech synthesis does in fact require more training and often presents, at the beginning, some difficulties in comprehension. However, this does not mean that the final result is less suitable or less effective.

Many view speech synthesis as a sort of "sonic Braille", i.e. a mere transduction of the same information, usually presented on a Braille display, into a audio modality, 
requiring the same approach for reading it. Also, the common name of "screen readers", used to indicate programs for accessing the computer through synthetic speech, appears inspired by this incorrect concept.

The term "screen reader" evokes the idea of a line by line presentation of the screen contents, like Braille displays generally do, and not of an intelligent selection of the various pieces of information according the needs and the class of the textual information, much like what is performed by the sophisticated speech program developed for the DOS environment in the last decade.

Incidentally, it should be observed that we utilize the name "screen reader" in the title of this paper only to use a generally accepted term, even if it appears much less adequate in Windows environment than in DOS.

In fact, the approach followed in developing the so-called "screen reader for Windows", in most cases, is not related to the graphic presentation of objects on the screen but on their logical concatenation, which is the relevant part of the information.

Only the access to the text displayed in an application window, such as a word processor, presents a problem similar to that of the corresponding application for DOS, but also in this case the "reading" does not necessarily consist of the line by line translation into voice.

\section{The "Off screen model"}

The GUIB project [1] approach to the problem of accessing MS Windows by blind persons is based on the reconstruction of the graphic user interface environment and the set of objects presented on the screen into a data structure, conceived in the form of a database, in which the relevant information is represented by the logical relationships among the elements, regardless their real graphic presentation. This is called "Off screen model" [2].

The database is automatically updated corresponding to the events of the environment through a system of filters contained in the runtime modules linked to the user interface. An appropriate language, called ERL (Event Response Language), has been developed by the project to collect, request, organize and provide the necessary information related to the events and the objects represented in the database.

A variety of solutions to the problem of effective access to this data structure have been developed by the project. The more general and effective solution is based on a multi-media presentation and interaction set consisting of integrating a large transitory Braille display, a speech synthesizer, a sound processor and a touch pad emulating the mouse.

Other less expensive solutions are under development to ensure different possibilities of access with a high level of effectiveness as well.

In this paper, a solution based only on the speech output is described, which is currently under development at IROE-CNR, in cooperation with a sub-contractor: 
IDEA-I.S.E. We will utilize the experience of the development of a screen reader for DOS, called Parla [3], which is one of the most diffused in Italy.

\section{The screen reader for Windows}

Two operating phases are provided for the screen reader: a "Tracking" phase, in which a real time voice feedback is produced in relation to the keyboard or mouse actions, and a "Review" phase, in which a large number of commands are available for an off line exploration of the environment and to set a number of flags and parameters for the speech synthesizer and the screen reader itself.

A "hot key" allows switching between these two phases.

This organization is similar to that of the most sophisticated screen readers for DOS. Such a similarity also regards the interaction with the text contained in the "client area" of an application or the items of menus and listboxes.

In fact, in the Tracking phase, any cursor or selection bar movement is followed by the program and the character, word or line pointed to are automatically uttered, according the user's choices. This ensures control of any text editing operations or the selection of items in the other cases.

More specifically related to the problem of interaction with the software architecture of Windows environment are the possibilities of vocal feedback during the navigation through the tree of applications and the objects of the user interface such as buttons and dialogboxes. According to the user's choices, some pieces of information are automatically spoken in Tracking phase when one of these objects is selected or when a window is opened, under the action of a key or the mouse.

Also, runtime error or request messages for confirmation of a command can be uttered automatically.

The main differences with a screen reader for DOS concern the Review phase. Due to the complex environment and to its reconstruction in form of database, commands of exploration of such an environment are conceived to navigate along a number of directions inside the tree of objects, regardless the position of each object on the screen.

In other words, the database is considered to be a sort of hypertext distributed in a multi-dimensional space. This allows the blind user to realize the structure of the tree of objects much better than a trivial exploration of the graphic presentation in which the objects appear to be projected onto a two-dimensional space, and consequently their images often result overlapped.

Incidentally, we can observe that the analysis of the graphic layout would be very difficult without a panoramic vision.

In order to obtain an effective organization of the Review phase, it has been divided into four sub-environments: Navigation, Search, Text and Parameters. 
The Navigation sub-environment is devoted to the exploration of the tree and its elements. a number of couple of keys are devoted to move along the different directions. For example, in the tree: up/down (parent to first child and viceversa), right/left (among children), jump to the top, to the bottom, to the beginning or the end of the sequence of children; inside a dialog box: rotation among listboxes, comboboxes, edit-fields, buttons; in menus: horizontal and vertical lists of items.

Text environment is devoted to the exploration of a text, line by line, word by word, character by character.

Search environment allows the user to search for a specified piece of information, such as a string inside a text, an item inside a menu or a listbox, the name of an application, a specified button etc..

Finally, Parameters Environment allows the user to select different operating modalities of the screen reader and of the speech synthesizer. A number of flags and switches are provided for these needs.

In addition, some special commands are available to produce artificial actions of the mouse, such as the movement up to a specified object, followed or not by a single or a double click. This represents the generalization of the "cursor routing" function provided from the screen readers for DOS and makes this screen reader for Windows a powerful tool for a blind person.

In fact, the possibility to replace the use of the mouse with artificial actions, controlled directly in Review phase, solves one of the main problems for the blind user in using the Windows interface. The mouse is not a suitable pointing device for a blind person because of the difficulty in realizing quickly the absolute position of its pointer on the screen. Usually, a blind person prefers to perform the same actions by means of key strokes. The real-time vocal feedback ensured in Tracking phase allows to follow the effect of the various keys for the interaction with the user interface.

The "generalized cursor routing" allows the user to obtain the same results with an alternative procedure, which can be more effective than the sequence of keystrokes in the Tracking phase.

In fact, this utilizes the powerful features of exploration of the Review phase in order to understand the logical layout, to individuate the appropriate action and to produce it directly, avoiding the repetition of a sequence of actions in Tracking phase.

We can observe that, in most of the interaction acts, the user does not know how or where an object is displayed on the screen. Even in the case of menus, the concepts of "horizontal" and "vertical" are not important. The blind user is free to imagine the environment in a different spatial distribution. The same occurs when a text is read, if the user is only interested in the content. (When we are listening to a radio-news or to a "talking book" recorded on cassettes, we do not relate what we hear to the written text that the speaker sees).

Only in some specific cases does the spatial distribution of a text result important, and the user needs to be able to perceive such a distribution. For example, in a spreadsheet 
or in a word processor, it can be necessary to verify the format of the page.

Usually, this is the case in which a Braille display results more effective than a speech output. However, the potential disadvantage of the speech can be compensated by appropriate functions to transform the spatial position into different pitches or even by producing a sophisticated stereo-phonic effect [4].

\section{Conclusions}

The possibility of an effective man-machine interaction based only on the synthetic speech as an alternative display for the blind has been demonstrated in textual presentations in DOS environment. A large number of blind persons successfully utilize the personal computer with speech output.

The new challenge is the possibility of a similar approach to the graphic user interfaces.

In this paper we have presented a potentially satisfactory solution which requires careful evaluation and probably some additional development. However, the first results appear encouraging.

This opens good perspectives for an effective and rather inexpensive solution to the problem of new barriers produced by the technical development in the computer field.

\section{Acknowledgement}

This work has been supported by the GUIB project (Textual and Graphic User Interfaces for Blind persons), in the framework of the EEC program TIDE (Technology Initiatives for Disabled and Elderly people).

\section{References}

1 Emiliani P.L. "Graphical User Interfaces for Blind People"; in Proccedings of ECART2 Conference, Stockholm, Sweden, ISBN 918833619 0, 1993.

2 Kochanek D.: "Designing an OffScreen Model for a GUI"; in Proceedings of the 4th ICCHP, Vienna 1994.

3 P. Graziani: "Programma PARLA: Ausilio tiflotecnico per l'accesso al personal computer IBM-compatibile per mezzo della sintesi vocale", Report TR/ESI/08.93, IROE-CNR, Firenze 1993 (Italian language).

4 Crispien K., Würz W., Weber G.: "Using Spatial Audio for the Enhanced Presentation of Synthesised Speech within Screen-Readers for Blind Computer Users"; in Proceedings of the 4th ICCHP, Vienna 1994. 